\title{
Comparison of overall survival of gastric neoplasms containing neuroendocrine carcinoma components with gastric adenocarcinoma: a propensity score matching study
}

\author{
Jiahui Chen ${ }^{\dagger}$, Anqiang Wang ${ }^{\dagger}, \mathrm{Ke} \mathrm{Ji}^{\dagger}$, Zhaode Bu ${ }^{*}$ and Jiafu Ji ${ }^{*}$
}

\begin{abstract}
Background: Gastric neoplasms containing neuroendocrine carcinoma (NEC) components are rare malignancies with highly aggressive behavior and a poor prognosis and include pure NEC and mixed tumors containing NEC components. We aimed to investigate whether there is a distinct difference in overall survival (OS) between gastric neoplasms containing NEC components and gastric adenocarcinoma.

Methods: Surgically resected gastric neoplasms containing NEC components $(n=180)$ and gastric adenocarcinomas $(n=785)$ from January 2013 to December 2019 at Peking University Cancer Hospital were retrospectively analysed. Patients were categorized into a surgical group and a neoadjuvant group and adjusted using propensity score matching. In the two groups, gastric neoplasms containing NEC components were divided into pure NEC and mixed tumors with less than 30\% (<30\% G-HMiNEN), between 30 and 70\% (G-HMiNEN) and more than $70 \%$ (> 70\% G-HMiNEN) neuroendocrine carcinoma components. OS was compared between these groups and the gastric adenocarcinoma group.
\end{abstract}

Results: The OS of gastric neoplasms containing neuroendocrine NEC components was poorer than that of gastric adenocarcinomas in the surgical group, regardless of whether the percentage of neuroendocrine cancer components was less than $30 \%$, between 30 and $70 \%$, more than $70 \%$ or $100 \%$. Cox multivariable regression analysis suggested that tumor category (neoplasms containing NEC components or gastric adenocarcinoma) was an independent risk factor for prognosis. Interestingly, among patients receiving neoadjuvant therapy, the difference was not significant.

(Continued on next page)

\footnotetext{
* Correspondence: buzhaode@cjcrcn.org; ijijafu@hsc.pku.edu.cn

${ }^{\dagger}$ Jiahui Chen, Anqiang Wang and Ke Ji contributed equally to this work. Department of Gastrointestinal Surgery, Key Laboratory of Carcinogenesis and Translational Research (Ministry of Education), Peking University Cancer Hospital \& Institute, No. 52 Fucheng Road, Haidian District, Beijing 100142, China
}

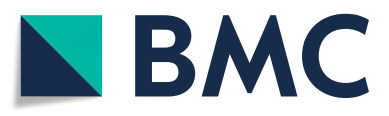

(c) The Author(s). 2020 Open Access This article is licensed under a Creative Commons Attribution 4.0 International License, which permits use, sharing, adaptation, distribution and reproduction in any medium or format, as long as you give appropriate credit to the original author(s) and the source, provide a link to the Creative Commons licence, and indicate if changes were made. The images or other third party material in this article are included in the article's Creative Commons licence, unless indicated otherwise in a credit line to the material. If material is not included in the article's Creative Commons licence and your intended use is not permitted by statutory regulation or exceeds the permitted use, you will need to obtain permission directly from the copyright holder. To view a copy of this licence, visit http://creativecommons.org/licenses/by/4.0/. The Creative Commons Public Domain Dedication waiver (http://creativecommons.org/publicdomain/zero/1.0/) applies to the data made available in this article, unless otherwise stated in a credit line to the data. 
(Continued from previous page)

Conclusions: Gastric neoplasms containing any proportion of NEC components had poorer overall survival than gastric adenocarcinoma in patients treated with surgery directly, indicating that these neoplasms are more malignant than gastric adenocarcinoma. Among the patients receiving neoadjuvant therapy, the difference in overall survival was not significant, which was in sharp contrast with the results of the surgery group, suggesting that neoadjuvant therapy may have a good effect in the treatment of these neoplasms.

Keywords: Neuroendocrine carcinoma, Gastric adenocarcinoma, Overall survival

\section{Background}

Gastric neoplasms containing neuroendocrine carcinoma (NEC) components are a heterogeneous subgroup of gastric cancer with highly aggressive behavior and poor prognosis and include pure NECs and mixed tumors containing NEC components. Every year there are approximately 1 million new cases of gastric cancer worldwide, and gastric neoplasms containing NEC components account for approximately $0.1-0.6 \%$ of these cases $[1,2]$. Given the low incidence, there is little comprehensive basic and clinical research to systematically guide the treatment of these gastric neoplasms, making the prognosis of these tumors unsatisfactory [3-7].

According to the 2017 World Health Organization (WHO) digestive neuroendocrine tumor classification, neuroendocrine neoplasm (NEN) can be divided into three categories based on Ki-67 levels and mitotic counts (× $10 \mathrm{HPF}$ ): Grade 1 (G1, Ki67 $\leq 2 \%$, mitoses $<2$ ), Grade $2(\mathrm{G} 2,3 \%<\mathrm{Ki} 67 \leq 20 \%, 2 \leq$ mitoses $\leq 20)$, Grade 3 (G3, Ki67 > 20\%, mitoses> 20) [8]. Meanwhile, the American Joint Committee on Cancer (AJCC) defines highly differentiated NEN as a neuroendocrine tumor (NET) and the poorly differentiated NEN as a neuroendocrine carcinoma (NEC) based on the degree of tumor cell differentiation. Generally, G1, G2, and rare well-differentiated G3 NENs belong to the NETs, while poorly differentiated G3 NENs belong to NECs [8, 9]. Gastric mixed neuroendocrine-non-neuroendocrine neoplasm (GMiNEN) is a special type of gastric NEN that is defined as containing more than $30 \%$ of both neuroendocrine and non-neuroendocrine components [8], accounting for approximately $7 \%$ of all G-NENs and $25 \%$ of gastric neuroendocrine carcinomas (G-NECs) [4-6]. For those mixed tumors with less than $30 \%$ or more than $70 \%$ neuroendocrine carcinoma components, there is no uniform definition. Considering the heterogeneity of MiNEN and the malignancy degree of the different components in the tumor, La Rosa et al. [10, 11] proposed dividing MiNEN into three categories: high-grade, intermediate-grade and low-grade. High-grade MiNEN consists of NEC and carcinoma/adenoma, intermediategrade MiMEN consists of NET and carcinoma, and lowgrade MiNEN consists of NET and adenoma. Therefore, in this study, gastric high-grade mixed neuroendocrine- non-neuroendocrine neoplasm (G-HMiNEN) was defined as gastric cancer containing more than $30 \%$ of both neuroendocrine carcinoma and adenocarcinoma components.

Generally, the prognosis of mixed tumors is largely determined by the most malignant component. Kim et al. [12] found that G-NEC has shorter progression-free survival (PFS) than gastric adenocarcinoma. Huang et al. [13] found that the prognosis of patients with more than $50 \%$ of neuroendocrine cancer components is significantly poorer than that of patients with less than $50 \%$ components. All of these studies provide evidence that tumors containing neuroendocrine cancer components may contribute to a worse prognosis. Therefore, we hypothesized that a mixed tumor containing neuroendocrine carcinoma components would have a worse prognosis than pure adenocarcinoma alone. We sought to find studies on the overall survival (OS) comparison between G-HMiNEN and gastric adenocarcinoma but failed. Thus, we think that a study of the comparison of the OS of G-HMiNEN and gastric adenocarcinoma will provide a valuable supplement to current research on GHMiNEN. To overcome the bias caused by the differences between the covariates in the comparison, we used propensity score matching (PSM) to match important factors such as age, gender, tumor location, tumor size, pathological staging, and adjuvant chemotherapy between the two groups, making the research results more reliable.

\section{Methods}

\section{Patient selection}

We retrospectively collected patients diagnosed with gastric NENs and underwent radical resection at Peking University Cancer Hospital, Beijing, from January 2013 to December 2019. The inclusion criteria were as follows: (1) pathologically confirmed pure NEC or tumor containing NEC components; (2) no other tumors were diagnosed before the operation; (3) complete clinicopathological information and survival information that could be obtained through follow-up. Patients diagnosed with cM1 or cT4b before surgery or died from perioperative complications were excluded from the study. 
Patients with gastric adenocarcinomas undergoing radical surgery were randomly selected for PSM analyses.

\section{Follow-up}

We followed the patients at least twice a year. Serum tumor markers test, gastroscope, and computed tomography $(\mathrm{CT})$ scans were used to reexamine patients after surgery. Depending on the patients' status, Magnetic resonance imaging (MRI) and Positron emission tomography \& computed tomography (PET-CT) were also considered. For patients who cannot regularly visit our center for postoperative examination, we use telephone follow-up to obtain survival information.

\section{Diagnosis and classification}

We re-evaluated the diagnosis and classification of GHMiNEN. Mixed tumors with less than $30 \%$ or more than $70 \%$ neuroendocrine carcinoma components were also included in this study, which were defined as $<30 \%$ G-HMiNEN and $>70 \%$ G-HMiNEN, respectively. A tumor consisting of $100 \%$ NEC is defined as pure NEC. All neuroendocrine tumors were identified, diagnosed, and classified by two independent pathologists in accordance with the 2017 WHO classification of tumors [8]. Neuroendocrine components were identified by histological features and immunohistochemical specificity marks, such as synaptophysin (Syn), chromogranin A (CgA), and neuro cell adhesion molecule (CD56 or NCAM). The tumor staging described in the study was based on the AJCC 8th Edition TNM Staging Guidelines [9]. All possible disagreements were discussed in our study group.

\section{Definition of variables and groups}

In this study, patients were divided into a surgical group and a neoadjuvant group based on whether they had received neoadjuvant therapy before surgery. Patients in the surgery group were assessed by the pTNM staging system, while patients in the neoadjuvant treatment group were assessed by the ypTNM staging system. OS refers to the time from surgery to the last follow-up, the time of death, or the end of follow-up (e.g., loss of follow-up or other cause of death).

\section{Propensity score matching}

To accurately compare the prognosis of G-HMiNEN and gastric adenocarcinoma, we employed PSM to balance the differences between the two groups. PSM was performed through the Pamatching 3.04 plug-in in SPSS 22.0 software. Logistic regression models were used to estimate propensity scores based on gender, age, tumor location, tumor size, and pathological staging. Given a 0.2 caliper width, 1:2 nearest neighbor matching was performed. The chi-squared test and Mann-Whitney U test were used to further verify the matching results.

\section{Statistical analysis}

All statistical analyses were performed using SPSS 22.0 statistical software (IBM, United States). The chisquared test and Mann-Whitney U test were used for statistical analysis of categorical variables and continuous variables, respectively. Kaplan-Meier method was used for the comparison of OS. The log-rank test was used to compare survival rates. Multivariable Cox proportional hazards models were used to identify predictors of survival outcome. $P<0.05$ was regarded as the threshold of significance.

\section{Results}

\section{Patient selection and PSM results}

Between 2013 and 2019, among the patients treated at the Gastrointestinal Cancer Center of Peking University Cancer Hospital, a total of 180 patients with gastric neoplasms containing NEC components met the inclusion criteria for the study, including 55 cases of pure NEC and 125 cases of mixed-type. Of these patients, a total of 65 patients received neoadjuvant therapy (NEC: 27, > 70\% G-HMiNEN: 5, G-HMiNEN: 19, <30\% GHMiNEN: 12), while the remaining 117 patients received surgery directly (NEC: 28, >70\% G-HMiNEN: 8 , GHMiNEN: $43,<30 \%$ G-HMiNEN: 38). There were an insufficient number of patients in group $>70 \%$ GHMiNEN group to conduct effective statistical analysis, so we combined the $>70 \%$ G-HMiNEN group with the NEC group for further analysis. We also randomly selected 785 patients with gastric adenocarcinoma who underwent radical surgery. Among them, 477 patients received neoadjuvant therapy, and the remaining $308 \mathrm{pa}-$ tients were treated with surgery directly (Fig. 1).

Immunohistochemical specificity markers were utilized to identify the neuroendocrine components (Fig. 2a). Syn was expressed in almost all neoplasms containing NEC components (98.3\%), while the positive rates of CgA and CD56 were much lower (62.8 and 66.7\%, respectively). No significant difference in the positive rate of Syn and CgA was observed between pure NEC, > 70\% G-HMiNEN, G-HMiNEN, and $<30 \%$ G-HMiNEN (Fig. 2b, c), only the positive rate of CD56 was found to be higher in the pure NEC group than that in the $<30 \%$ G-HMiNEN group (Fig. 2d).

Therefore, prior to OS comparison, PSM was performed to ensure that there were no significant differences in patient gender, age, tumor location, tumor size, pathological staging, and adjuvant chemotherapy between the two groups. 


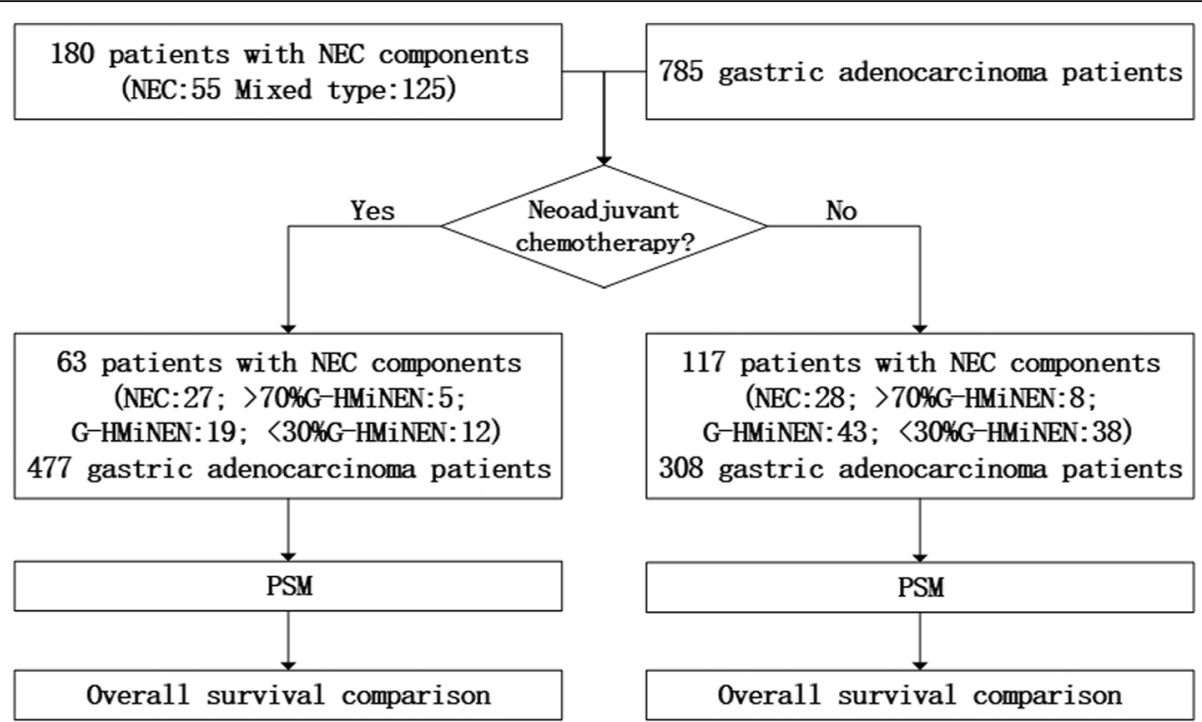

Fig. 1 Flow chart of patient enrolment

Comparison of OS between all patients with NEC components and patients with gastric adenocarcinoma in the surgical group and neoadjuvant group

Before PSM, we compared the survival curves between all patients with NEC components and patients with gastric adenocarcinoma by the Kaplan-Meier method (Fig. 3). Apparently, patients with NEC components had a poorer OS than those with gastric adenocarcinoma (Fig. 3a, $p<0.0001$ ) in the surgical group. In contrast, no significant difference was observed between the patients receiving neoadjuvant therapy (Fig. 3b, $p=0.1467$ ). According to the proportion of NEC components, patients were classified into pure NEC, >70\% G-HMiNEN, G-HMiNEN, and $<30 \%$ G-HMiNEN. The OS was also compared between patients with adenocarcinoma and these groups, and the results were similar to the overall comparison (Fig. 3c, d).

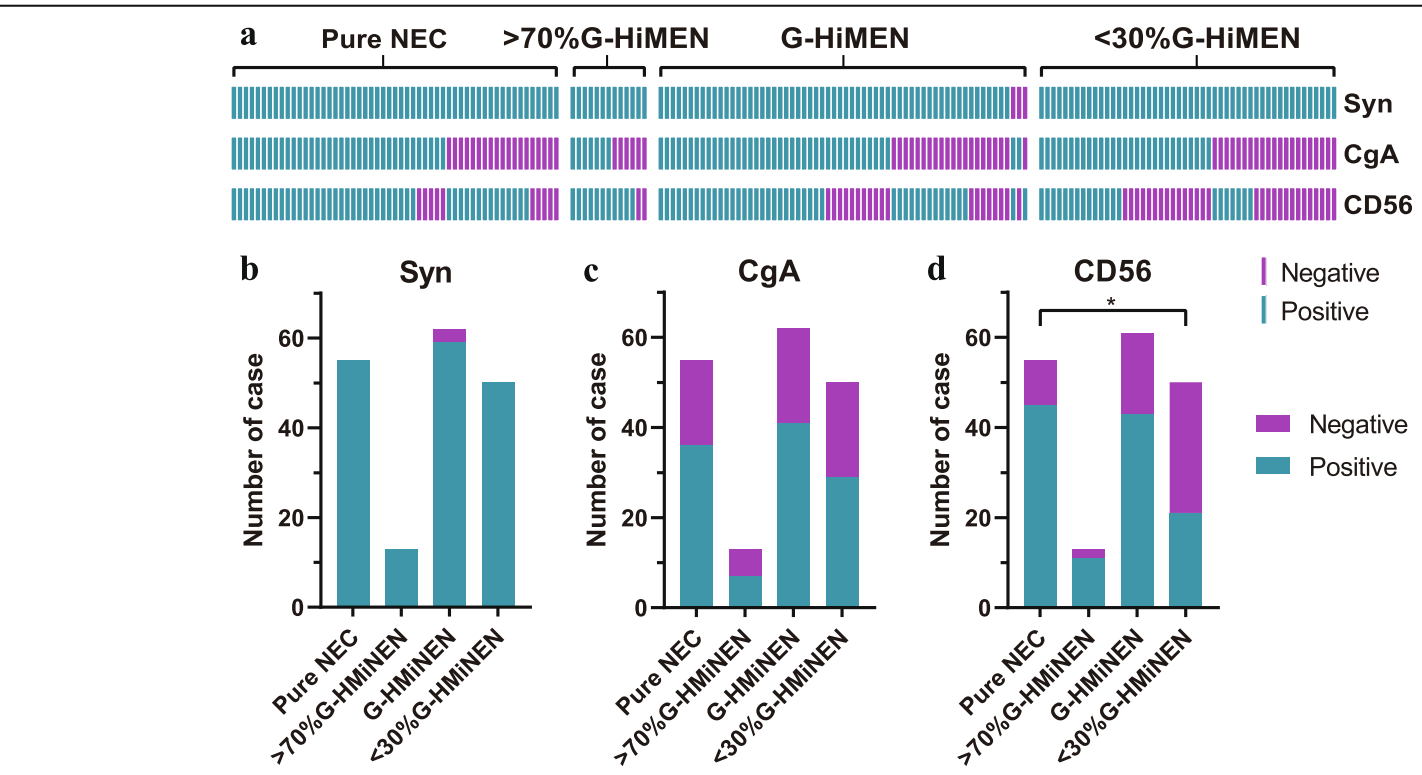

Fig. 2 Illustrations of immunohistochemical staining patterns in gastric neoplasms containing NEC components. a. An overview of the expression of Syn, CgA, and CD56 in tumors containing NEC components. b. Syn expression in different NEC component groups. c. CgA expression in different NEC component groups. d. CD56 expression in different NEC component groups. CD56, neuro cell adhesion molecule; CgA, chromogranin A; NEC, neuroendocrine carcinoma; Syn, synaptophysin; $P$-value $<0.05\left(^{*}\right)$ 


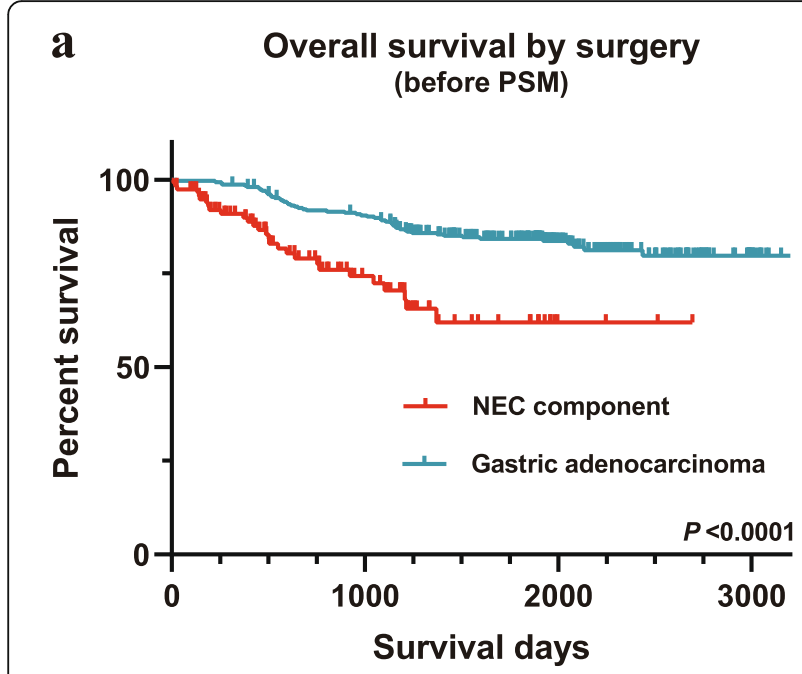

b Overall survival by neo-adjuvant therapy

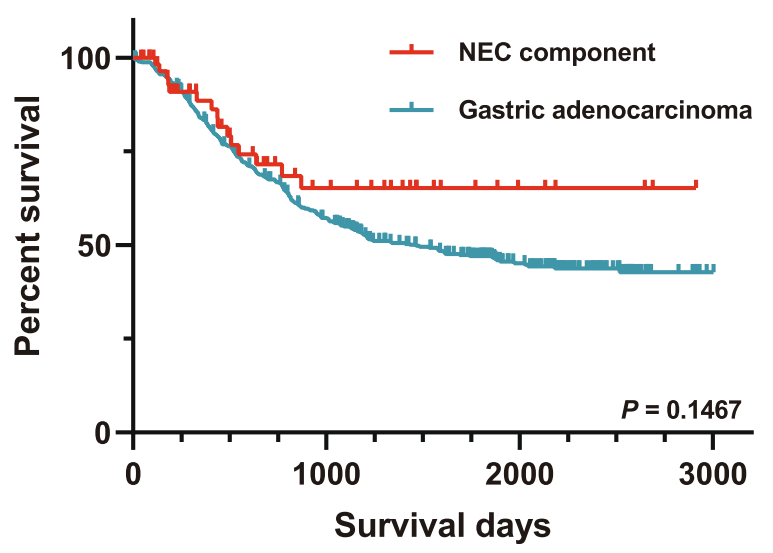

C Overall survival by surgery

d Overall survival by neo-adjuvant therapy (before PSM) (before PSM)

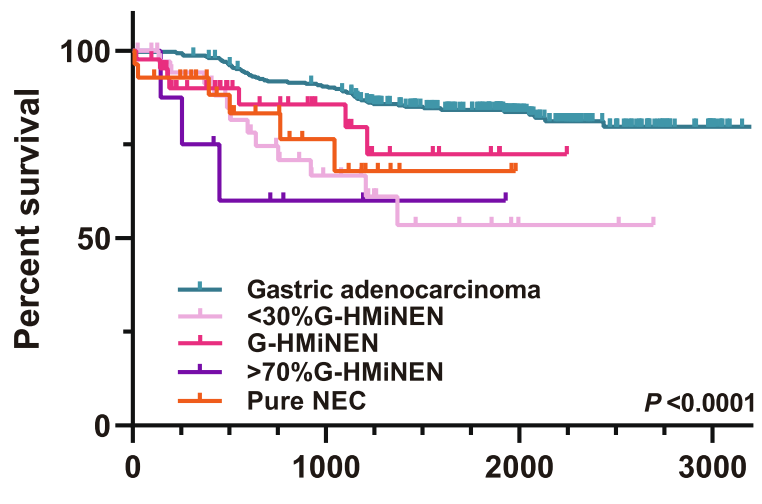

Survival days

e Overall survival by surgery (after PSM)

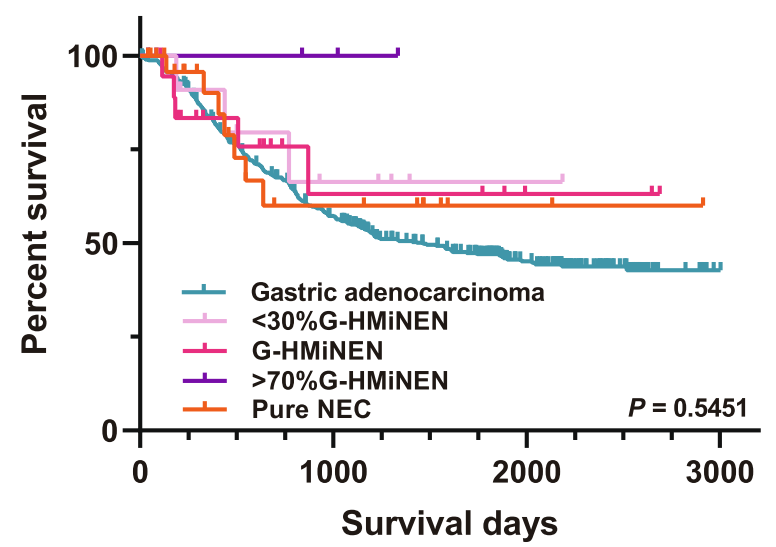

f Overall survival by neo-adjuvant therapy (after PSM)

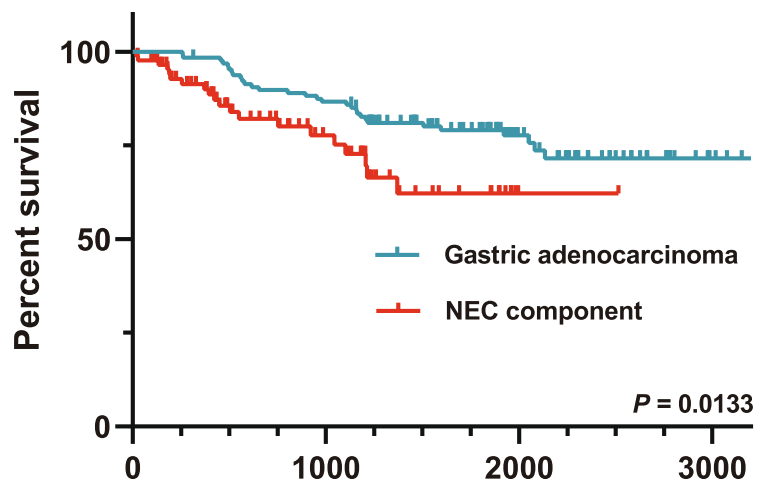

Survival days

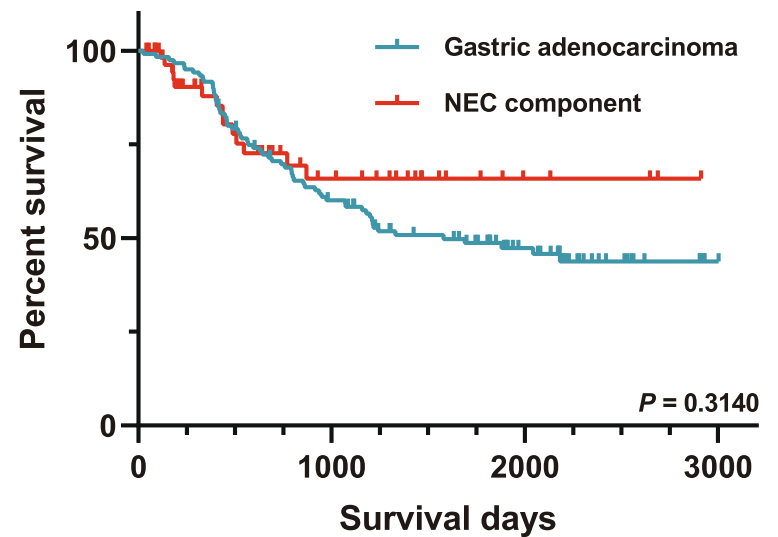

Fig. 3 (See legend on next page.) 
(See figure on previous page.)

Fig. 3 Comparison of OS between gastric neoplasms containing NEC components and gastric adenocarcinoma. a. OS comparison between gastric neoplasms containing NEC components and gastric adenocarcinoma before PSM in the surgical group. $\mathbf{b}$. OS comparison between gastric neoplasms containing NEC components and gastric adenocarcinoma before PSM in the neoadjuvant group. $\mathbf{c}$. OS comparison between different NEC content groups (pure NEC, > 70\% G-HMiNEN, G-HMiNEN, and < 30\% G-HMiNEN) and gastric adenocarcinoma before PSM in the surgical group. d. OS comparison between the different NEC content groups and gastric adenocarcinoma before PSM in the neoadjuvant group. e. OS comparison for patients in the surgical group after PSM. f. OS comparison for patients in the neoadjuvant group after PSM. NEC, neuroendocrine carcinoma; OS, overall survival; PSM, propensity score matching

Before PSM, significant differences between the baseline characteristics were observed in the surgical group and the neoadjuvant group (Table $1 \&$ Table 2). To balance the clinicopathological differences between the two groups, PSM was performed to ensure that there were no significant differences in patient gender, age, tumor location, tumor size, pathological staging, and adjuvant chemotherapy between the two groups. The detailed clinicopathological characteristics before and after PSM are shown in Table 1 and Table 2.

As a result, 88 patients with NEC components and 128 patients with gastric adenocarcinoma were matched in the surgical group (Table 1). Patients with NEC components also had a poorer OS than those with gastric adenocarcinoma (Fig. 3e, $p=0.0133$ ). Multivariable analysis showed that adjuvant therapy, tumor category, and TNM stage were independent prognostic factors (Table 3).

To investigate whether neoadjuvant therapy had an effect on OS, 60 patients with NEC components and 120 patients with gastric adenocarcinoma were matched in the neoadjuvant group (Table 2). Interestingly, KaplanMeier analysis showed that among patients receiving neoadjuvant therapy, there was still no significant difference in OS between the two groups (Fig. 3f, $p=0.3140$ ).

\section{Comparison of OS between patients with different proportions of NEC components and patients with gastric adenocarcinoma}

To investigate whether the level of NEC components had an effect on OS in the surgical group, $<30 \%$ GHMiNEN, G-HMiNEN, pure NEC, and pure NEC plus $>70 \%$ G-HMiNEN were compared with gastric adenocarcinoma after PSM. The results showed that even the group with the lowest proportion of NEC components, the $<30 \%$ G-HMiNEN group, had a poorer OS than adenocarcinoma (Fig. 4a, $P=0.0130$ ). As expected, the G-HMiNEN, pure NEC, and pure NEC plus $>70 \%$ GHMiNEN groups, each with relatively high proportions of NEC components, had worse OS than the gastric adenocarcinoma group (Fig. 4b-d, $P=0.0271,0.0174$, 0.0310). Detailed clinical information after matching is shown in Additional file 1: Tables S1-S4.

PSM was also performed in the neoadjuvant group. In contrast to the results of the surgery group, in the pure
NEC group (containing the highest proportion of NEC component), there was still no significant difference in OS from gastric adenocarcinoma (Fig. $5 \mathrm{~d})$. The other three groups with lower NEC content were also not significantly different from gastric adenocarcinoma in terms of OS (Fig. 5a-c). Detailed clinicopathological characteristics before and after PSM are shown in Additional file 1: Tables S5-S8.

\section{Discussion}

Among gastric neuroendocrine neoplasms, the tumor containing NEC components is a special type, including pure NEC and mixed tumor containing NEC components. The incidence of these tumors is extremely low, but they are more invasive and have a poorer prognosis than well-differentiated G-NENs $[4,5]$.

In previous study, Kim et al. found that in patients who had not received neoadjuvant chemotherapy, progression-free survival (PFS) of pure G-NEC was poorer than that of gastric adenocarcinoma, while the PFS of mixed-type tumors was not significantly different from that of gastric adenocarcinoma [12]. In Kim's study, the mixed type was defined as NET mixed with gastric cancer, rather than NEC. NET is much less malignant than NEC $[14,15]$. This may be the reason why there was no significant difference in OS between mixed type and gastric adenocarcinomas. In addition, mixed tumors with less than $30 \%$ or more than $70 \%$ of NEC components were not included in that study, which we believe was a deficit of the study. PFS is an important indicator for evaluating prognosis, in many cases, it can reflect the trend of OS. Based on Kim's research results, we regarded tumors containing NEC components as a whole and found that the OS of these tumors was poorer than that of adenocarcinoma in the surgical group. In the comparison of OS between mixed tumors with different proportions of NEC components and gastric adenocarcinoma, the results for pure NEC cases was similar to Kim's. While the OS of mixed tumors was also poorer than that of gastric adenocarcinoma, whether the proportion of neuroendocrine cancer components was less than $30 \%$, between 30 and $70 \%$, or more than $70 \%$, which was not mentioned in Kim's study. Cox multivariable regression analysis showed that tumor category (neoplasm with NEC component or adenocarcinoma), 
Table 1 Comparison of clinicopathological characteristics before and after PSM in surgical group

\begin{tabular}{|c|c|c|c|c|c|c|}
\hline \multirow[t]{2}{*}{ Patient Characteristics } & \multicolumn{3}{|l|}{ Unmatched comparison } & \multicolumn{3}{|l|}{ Matched comparison } \\
\hline & $\begin{array}{l}\text { Patients with NEC } \\
\text { components }(n=117)\end{array}$ & $\begin{array}{l}\text { Gastric } \\
\text { adenocarcinoma } \\
(n=308)\end{array}$ & $P$ value & $\begin{array}{l}\text { Patients with NEC } \\
\text { components }(n=88)\end{array}$ & $\begin{array}{l}\text { Gastric } \\
\text { adenocarcinoma } \\
(n=128)\end{array}$ & $P$ value \\
\hline Age (year), mean $\pm S D$ & $61.7 \pm 9.4$ & $55.2 \pm 10.8$ & $<0.001$ & $59.7 \pm 9.4$ & $58.9 \pm 9.7$ & 0.551 \\
\hline Gender (male/female) & 100/17 & $282 / 26$ & 0.063 & $76 / 12$ & $112 / 16$ & 0.807 \\
\hline $\mathrm{BMI}$, mean $\pm \mathrm{SD}$ & $24.1 \pm 3.1$ & $23.6 \pm 3.6$ & 0.100 & $24.4 \pm 3.1$ & $23.8 \pm 3.6$ & 0.182 \\
\hline Adjuvant therapy & & & $<0.001$ & & & 0.189 \\
\hline Yes & $87(74.4)$ & $109(35.4)$ & & $58(65.9)$ & $73(57.0)$ & \\
\hline No & $30(25.6)$ & $199(64.6)$ & & $30(34.1)$ & $55(43.0)$ & \\
\hline Tumor location & & & $<0.001$ & & & 0.679 \\
\hline Upper third & 70 (59.8) & $74(24.0)$ & & $46(52.3)$ & $60(46.9)$ & \\
\hline Middle third & $18(15.4)$ & $41(13.3)$ & & $14(15.9)$ & $19(14.8)$ & \\
\hline Lower third & $28(23.9)$ & $192(62.3)$ & & $28(31.8)$ & $48(37.5)$ & \\
\hline Entire & $1(0.9)$ & $1(0.3)$ & & $0(0.0)$ & $1(0.8)$ & \\
\hline Tumor size & & & 0.023 & & & 0.669 \\
\hline$<5 \mathrm{~cm}$ & $80(68.4)$ & $243(78.9)$ & & $63(71.6)$ & $95(74.2)$ & \\
\hline$\geq 5 \mathrm{~cm}$ & 37 (31.6) & $65(21.1)$ & & $25(28.4)$ & $33(25.8)$ & \\
\hline Type of gastrectomy & & & $<0.001$ & & & 0.077 \\
\hline Total gastrectomy & $78(66.7)$ & 79 (25.6) & & $53(60.2)$ & $59(46.1)$ & \\
\hline Distal gastrectomy & $29(24.8)$ & $206(66.9)$ & & $29(33.0)$ & $51(39.8)$ & \\
\hline Proximal gastrectomy & $10(8.5)$ & $23(7.5)$ & & $6(6.8)$ & $18(14.1)$ & \\
\hline Surgical procedure & & & $<0.001$ & & & 0.001 \\
\hline Open & $106(90.6)$ & $212(68.6)$ & & $80(90.9)$ & $92(71.9)$ & \\
\hline Laparoscopic & $11(9.4)$ & $96(32.2)$ & & $8(9.1)$ & $36(28.1)$ & \\
\hline T stage & & & $<0.001$ & & & $<0.001$ \\
\hline $\mathrm{T} 1$ & $16(13.7)$ & $195(63.3)$ & & $14(15.9)$ & $53(41.4)$ & \\
\hline $\mathrm{T} 2$ & $26(22.2)$ & $18(5.8)$ & & $25(28.4)$ & $12(9.4)$ & \\
\hline T3 & $48(41.0)$ & 49 (15.9) & & $27(30.7)$ & $35(27.3)$ & \\
\hline $\mathrm{T} 4$ & $27(23.1)$ & $46(14.9)$ & & $22(25.0)$ & $28(21.9)$ & \\
\hline N stage & & & $<0.001$ & & & 0.428 \\
\hline No & $48(41.0)$ & $210(68.2)$ & & $37(42.0)$ & $62(48.4)$ & \\
\hline N1 & $32(27.4)$ & $26(8.4)$ & & $22(25.0)$ & $22(17.2)$ & \\
\hline N2 & $20(17.1)$ & $27(8.8)$ & & $16(18.2)$ & $16(18.2)$ & \\
\hline N3 & $17(14.5)$ & 45 (14.6) & & $13(14.8)$ & $24(18.8)$ & \\
\hline M stage & & & 0.216 & & & 0.406 \\
\hline Mo & $117(100.0)$ & $304(98.7)$ & & $88(100.0)$ & $127(99.2)$ & \\
\hline M1 & $0(0.0)$ & $4(1.3)$ & & $0(0.0)$ & $1(0.8)$ & \\
\hline pTNM stage & & & $<0.001$ & & & 0.399 \\
\hline 1 & $30(25.6)$ & $202(65.6)$ & & $30(34.1)$ & $56(43.8)$ & \\
\hline$\|$ & $47(40.2)$ & $32(10.4)$ & & $26(29.5)$ & $30(23.4)$ & \\
\hline III & $40(34.2)$ & $70(22.7)$ & & $32(36.4)$ & $41(32.0)$ & \\
\hline IV & $0(0.0)$ & $4(1.3)$ & & $0(0.0)$ & $1(0.8)$ & \\
\hline
\end{tabular}


Table 2 Comparison of clinicopathological characteristics before and after PSM in neoadjuvant group

\begin{tabular}{|c|c|c|c|c|c|c|}
\hline \multirow[t]{2}{*}{ Patient Characteristics } & \multicolumn{3}{|l|}{ Unmatched comparison } & \multicolumn{3}{|l|}{ Matched comparison } \\
\hline & $\begin{array}{l}\text { Patients with NEC } \\
\text { components }(n=63)\end{array}$ & $\begin{array}{l}\text { Gastric } \\
\text { adenocarcinoma } \\
(n=477)\end{array}$ & $P$ value & $\begin{array}{l}\text { Patients with NEC } \\
\text { components }(n=60)\end{array}$ & $\begin{array}{l}\text { Gastric } \\
\text { adenocarcinoma } \\
(n=120)\end{array}$ & $P$ value \\
\hline Age (year), mean \pm SD & $61.2 \pm 9.6$ & $58.3 \pm 10.3$ & 0.037 & $61.3 \pm 9.7$ & $60.2 \pm 9.5$ & 0.484 \\
\hline Gender (male/female) & $52 / 11$ & $367 / 110$ & 0.316 & $50 / 10$ & $104 / 16$ & 0.549 \\
\hline BMI, mean $\pm S D$ & $24.1 \pm 3.9$ & $23.5 \pm 3.4$ & 0.285 & $23.9 \pm 3.9$ & $23.8 \pm 3.4$ & 0.855 \\
\hline Adjuvant therapy & & & 0.124 & & & 0.173 \\
\hline Yes & $58(92.1)$ & $459(96.2)$ & & $56(93.9)$ & $117(97.5)$ & \\
\hline No & $5(7.9)$ & $18(3.8)$ & & $4(6.7)$ & $3(2.5)$ & \\
\hline Tumor location & & & $<0.001$ & & & 0.361 \\
\hline Upper third & $46(73.0)$ & $222(46.5)$ & & $43(71.7)$ & $79(65.8)$ & \\
\hline Middle third & $9(14.3)$ & $50(10.5)$ & & $9(15.0)$ & $13(10.8)$ & \\
\hline Lower third & $7(11.1)$ & 189 (39.6) & & $7(11.7)$ & $21(17.5)$ & \\
\hline Entire & $1(1.6)$ & $16(3.4)$ & & $1(1.7)$ & $7(5.8)$ & \\
\hline Tumor size & & & 0.325 & & & 0.594 \\
\hline$<5 \mathrm{~cm}$ & $36(57.1)$ & $303(63.5)$ & & $33(55.0)$ & $71(59.2)$ & \\
\hline$\geq 5 \mathrm{~cm}$ & $27(42.9)$ & $174(36.5)$ & & $27(45.0)$ & $49(40.8)$ & \\
\hline Type of gastrectomy & & & $<0.001$ & & & 0.974 \\
\hline Total gastrectomy & $50(79.4)$ & $239(50.1)$ & & 47 (78.3) & $94(78.3)$ & \\
\hline Distal gastrectomy & $7(11.1)$ & 181 (37.9) & & $7(11.7)$ & $15(12.5)$ & \\
\hline Proximal gastrectomy & $6(9.5)$ & $57(11.9)$ & & $6(10.0)$ & $11(9.2)$ & \\
\hline Surgical procedure & & & 0.394 & & & 1.000 \\
\hline Open & $60(95.2)$ & $440(92.2)$ & & $57(95.0)$ & $114(95.0)$ & \\
\hline Laparoscopic & $3(4.8)$ & $37(7.8)$ & & $3(5.0)$ & $6(5.0)$ & \\
\hline T stage & & & $<0.001$ & & & 0.065 \\
\hline T0 & $1(1.6)$ & $0(0.0)$ & & $0(0.0)$ & $0(0.0)$ & \\
\hline $\mathrm{T} 1$ & $3(4.8)$ & $28(5.9)$ & & $3(5.0)$ & $3(2.5)$ & \\
\hline $\mathrm{T} 2$ & $3(4.8)$ & 60 (12.6) & & $3(5.0)$ & 15 (12.5) & \\
\hline $\mathrm{T} 3$ & 35 (55.6) & $154(32.3)$ & & $34(56.7)$ & 47 (39.2) & \\
\hline T4 & $21(33.3)$ & 235 (49.6) & & $20(33.3)$ & $55(45.8)$ & \\
\hline N stage & & & 0.017 & & & 0.186 \\
\hline NO & $17(27.0)$ & $158(33.1)$ & & $16(26.7)$ & 44 (36.7) & \\
\hline N1 & $22(34.9)$ & 99 (20.8) & & $21(35.0)$ & $33(27.5)$ & \\
\hline N2 & $14(22.2)$ & $80(16.8)$ & & $14(23.3)$ & $17(14.2)$ & \\
\hline N3 & $10(15.9)$ & $140(29.4)$ & & $9(15.0)$ & $26(21.7)$ & \\
\hline M stage & & & 0.041 & & & 0.721 \\
\hline MO & $60(95.2)$ & $471(98.7)$ & & 59 (98.3) & $117(97.5)$ & \\
\hline M1 & $3(4.8)$ & $6(1.3)$ & & $1(1.7)$ & $3(2.5)$ & \\
\hline ypTNM stage & & & 0.001 & & & 0.950 \\
\hline 0 & $1(1.6)$ & $0(0.0)$ & & $0(0.0)$ & $0(0.0)$ & \\
\hline I & $4(6.3)$ & $52(10.9)$ & & $4(6.7)$ & $6(5.0)$ & \\
\hline$\|$ & $31(49.2)$ & $163(34.2)$ & & $31(51.7)$ & $64(53.3)$ & \\
\hline III & $24(38.1)$ & $256(53.7)$ & & $24(40.0)$ & $47(39.2)$ & \\
\hline IV & $3(4.8)$ & $6(1.3)$ & & $1(1.7)$ & $3(2.5)$ & \\
\hline
\end{tabular}


Table 3 Univariate and multivariate analyses of survival after PSM in surgical group

\begin{tabular}{|c|c|c|c|c|c|c|}
\hline \multirow[t]{2}{*}{ Patient Characteristics } & \multicolumn{3}{|c|}{ Univariate analysis } & \multicolumn{3}{|c|}{ Multivariate analysis } \\
\hline & $\mathrm{HR}$ & $95 \% \mathrm{Cl}$ & $P$ value & $\mathrm{HR}$ & $95 \% \mathrm{Cl}$ & $P$ value \\
\hline Age (year) & 0.993 & $0.966-1.021$ & 0.608 & & & \\
\hline \multicolumn{7}{|l|}{ Gender } \\
\hline male vs. female & 0.542 & $0.271-1.084$ & 0.083 & & & \\
\hline $\mathrm{BMI}$ & 0.989 & $0.915-1.069$ & 0.782 & & & \\
\hline \multicolumn{7}{|l|}{ Adjuvant therapy } \\
\hline Yes vs. No & 9.490 & $3.411-26.400$ & $<0.001$ & 23.434 & $2.348-222.841$ & 0.007 \\
\hline \multicolumn{7}{|l|}{ Tumor size } \\
\hline$\geq 5 \mathrm{~cm}$ vs. $<5 \mathrm{~cm}$ & 2.557 & $1.458-4.485$ & 0.001 & 1.318 & $0.710-2.448$ & 0.381 \\
\hline \multicolumn{7}{|l|}{ Tumor category } \\
\hline $\begin{array}{l}\text { Carcinoma with NEC component vs. } \\
\text { Gastric adenocarcinoma vs. }\end{array}$ & 2.067 & $1.150-3.715$ & 0.015 & 1.955 & $1.050-3.642$ & 0.035 \\
\hline Type of gastrectomy & & & 0.021 & & & 0.230 \\
\hline Total gastrectomy & 1 & 1 & - & 1 & 1 & - \\
\hline Distal gastrectomy & 0.382 & $0.193-0.758$ & 0.006 & 0.545 & $0.256-1.160$ & 0.115 \\
\hline Proximal gastrectomy & 0.643 & $0.268-1.543$ & 0.323 & 1.130 & $0.449-2.844$ & 0.795 \\
\hline Surgical procedure & & & 0.499 & & & \\
\hline Laparoscopic vs. Open & 0.959 & $0.491-1.874$ & 0.904 & & & \\
\hline TNM stage & & & $<0.001$ & & & 0.009 \\
\hline । & 1 & 1 & - & 1 & 1 & \\
\hline$\|$ & 3.678 & $1.254-10.756$ & 0.018 & 0.176 & $0.021-1.484$ & 0.110 \\
\hline III & 10.657 & $4.161-27.296$ & $<0.001$ & 0.487 & $0.062-3.802$ & 0.492 \\
\hline IV & 30.036 & $3.443-262.046$ & 0.002 & 2.477 & $0.139-44.100$ & 0.537 \\
\hline
\end{tabular}

tumor size, and TNM staging were independent risk factors for prognosis. This suggests that the prognosis of gastric neoplasms with NEC components is substantially different from that of gastric adenocarcinoma, and even a small percentage $(<30 \%)$ of NEC components can also impair prognosis, which challenges the current cut-off value of $30 \%$.

The proportion of each component that must theoretically be greater than 30\% was set in 1987 [16]. And since 2010, WHO has also adopted this standard to define MiNEN [16]. This largely avoids the overdiagnosis of MiNEN in tumors with only focal neuroendocrine marker expression and no corresponding morphological changes. In addition, it also prevents clinicians from dealing with these rare neoplasms too often without guidelines [15]. Nevertheless, it is now being questioned by an increasing number of scholars. The components in mixed tumors are not evenly distributed. For large tumors, the randomness of biopsy and postoperative pathological sampling causes the proportion of each component to fluctuate greatly, making it difficult to describe the proportion of each component precisely [15]. Park et al. compared the OS between tumors with more than $10 \%$ NEC components and gastric adenocarcinoma with or without less than $10 \%$ NEC, and they found that tumors with an NEC composition of more than $10 \%$ had a worse prognosis. This suggests that even a small proportion of malignant components can affect prognosis [3]. While in Park's study, for unknown reasons, the authors did not compare the prognosis of mixed tumors with NEC components less than $10 \%$ with gastric adenocarcinomas directly, nor did they compare all NEC-containing tumors as a whole with gastric adenocarcinoma, which we believe was a deficit of the study. In our study, we regarded tumors containing NEC components as a whole and found that the OS of these tumors was poorer than that of adenocarcinoma in the surgical group. In addition, we also found that the OS of mixed tumors with less than $30 \%$, between 30 and $70 \%$, more than $70 \%$ NEC components or pure NEC was worse than that of gastric adenocarcinoma. Analysis of immunohistochemical markers show that there was no significant difference in the positive rate of Syn and CgA between different NEC content groups, only the positive rate of CD56 was found to be higher in the pure NEC group than that in the $<30 \%$ G-HMiNEN group. The role of CD56 in the diagnosis of NEC is still controversial. However, Syn and CgA are two well-recognized 


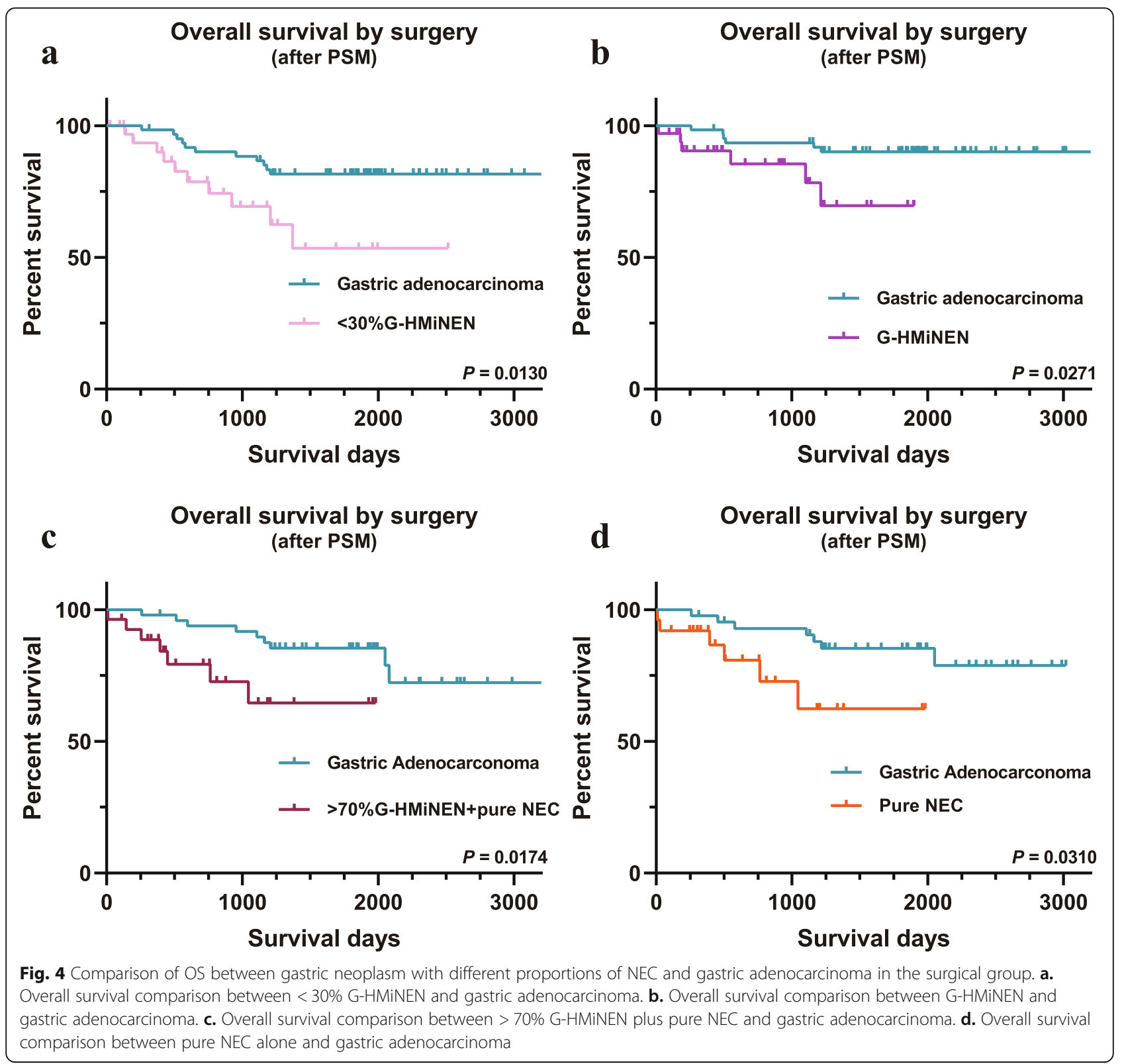

markers. Therefore, from the results of immunohistochemistry, we believed that there was no significantly difference in tumors containing NEC components. Studies on the molecular mechanism of pathogenesis show that NEC components and adenocarcinoma components have similar genomic abnormalities, similar losses of heterozygosity ( $\mathrm{LOH})$ and mutations at multiple loci and key oncogenes, such as TP53, $A P C$, and $R B$ genes. All these results imply that the two components in the mixed tumor may have a common origin and acquire biphenotypic differentiation during carcinogenesis [17-24]. Moreover, in the WHO definition of mixed neuroendocrine and non-neuroendocrine neoplasms of other organs (i.e., lung and thyroid) [25], no minimum percentage for either ingredient is established. Therefore, we believe that mixed tumors containing NEC components are actually of the same origin, have similar biological characteristics, and are different from gastric adenocarcinoma. We propose considering mixed tumors containing NEC components as a whole, rather than defining them based on the 30\% definition for both tumor components, which has not been raised by other studies.

Previously, many studies have confirmed the efficacy of neoadjuvant chemotherapy in gastric adenocarcinoma $[26,27]$. In a retrospective study involving 69 patients, $\mathrm{Ma}$ et al. found that neoadjuvant chemotherapy improves the survival of patients with NEC and HMiNEN of the stomach [28]. Van der Veen et al. reported that 


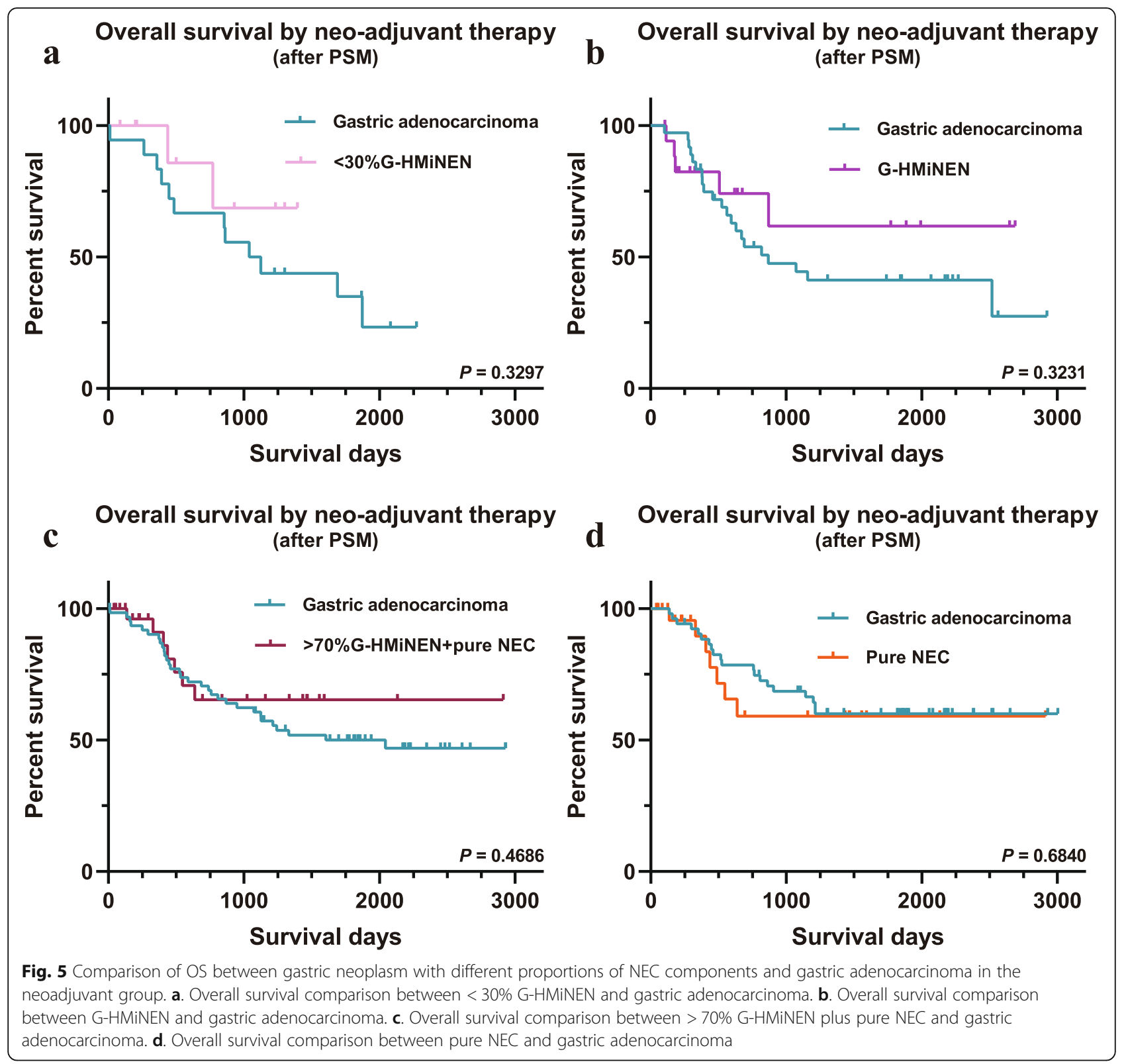

neoadjuvant chemotherapy could not benefit the survival of patients with mixed tumors containing NEC components [29]. However, because only eight patients were included in the neoadjuvant group, Van's results are questionable. In our study, among patients receiving neoadjuvant therapy, no significant difference in OS between mixed tumor and gastric adenocarcinoma was observed. Even for the pure NEC group with the highest NEC content, there was no significant difference, suggesting that neoadjuvant therapy may have a positive effect on these neoplasms.

Although this is only a single-center retrospective study, the sample we reported is considerable for this rare disease, which can provide new ideas for clinical and basic research. In addition, we proposed treating all gastric neoplasms containing NEC components as a whole and found that neoadjuvant therapy may have a good effect on these neoplasms. In the future, we will conduct more genomics studies to confirm our ideas. This study also has its limitations. Due to the lack of recurrence and detailed chemotherapy information, we were unable to compare progressionfree survival and analyse the effects of different chemotherapy regimens. As a retrospective study, despite our performing PSM in advance, selection bias cannot be completely avoided. In addition, since the exact proportion of each component in the mixed tumor could not be obtained, we could not determine 
whether there is a cutoff value for the diagnosis of the mixed tumor with NEC component less than $30 \%$, so we could only treat all mixed tumors with NEC component as a whole.

\section{Conclusions}

Our study demonstrated that gastric neoplasms with NEC components, regardless of the proportion of components, have poorer overall survival than gastric adenocarcinoma, indicating a higher degree of malignancy than gastric adenocarcinoma. Among the patients receiving neoadjuvant therapy, the difference in overall survival was not significant, which was in sharp contrast with the results of the surgery group, suggesting that neoadjuvant therapy may have a good effect on the prognosis of these malignancies. Therefore, for this type of malignancy, we should adopt more aggressive and powerful treatments than those used for gastric adenocarcinoma to improve the prognosis of patients. Neoadjuvant chemotherapy may be a good way to improve the efficacy of treatment for these tumors at advanced stages.

\section{Supplementary information}

Supplementary information accompanies this paper at https://doi.org/10. 1186/s12885-020-07281-7.

Additional file 1: Table S1. Comparison of clinicopathological characteristics before and after PSM of $<30 \%$ G-HMiNEN patients in surgical group. Table S2. Comparison of clinicopathological characteristics before and after PSM of G-HMiNEN patients in surgical group. Table S3. Comparison of clinicopathological characteristics before and after PSM of $>70 \%$ G-HMiNEN plus pure NEC patients in surgical group. Table S4. Comparison of clinicopathological characteristics before and after PSM of pure NEC patients in surgical group. Table S5. Comparison of clinicopathological characteristics before and after PSM of < 30\%G-HMiNEN patients in neoadjuvant group. Table S6. Comparison of clinicopathological characteristics before and after PSM of G-HMiNEN patients in neoadjuvant group. Table S7. Comparison of clinicopathological characteristics before and after PSM of $>70 \%$ G-HMiNEN plus pure NEC patients in neoadjuvant group. Table S8. Comparison of clinicopathological characteristics before and after PSM of pure NEC patients in neoadjuvant group.

\section{Abbreviations \\ AJCC: American Joint Committee on cancer; CT: Computed tomography; G- HMiNEN: Gastric high-grade mixed neuroendocrine-non-neuroendocrine neoplasm; G-NEC: Gastric neuroendocrine carcinoma; HPF: High power field; MiNEN: Mixed neuroendocrine-non-neuroendocrine neoplasm; NEC: Neuroendocrine carcinoma; NEN: Neuroendocrine neoplasm; NET: Neuroendocrine tumor; MRI: Magnetic resonance imaging; OS: Overall survival; PET-CT: Positron emission tomography \& computed tomography; PFS: Progression-free survival; PSM: Propensity score matching; WHO: World Health Organization}

\section{Acknowledgments}

Thanks to Dr. Zhongwu Li of the Department of Pathology, Peking University Cancer Hospital, and his colleagues for their assistance in pathological diagnosis and review. Thanks to all colleagues in the Department of Gastrointestinal Surgery of Peking University Cancer Hospital and Dr. Jiang Hong from the Statistics Department for their assistance in this study.

\section{Authors' contributions}

All authors contributed to the study conception and design. JC performed data collection and wrote the manuscript. AW wrote and t revised he manuscript. KJ helped with statistical analysis and prepared the illustrations. ZB edited the manuscript, JJ conceived the study and reviewed the manuscript. All authors read and approved the final manuscript.

\section{Funding}

This work was supported by the National Science Foundation for Young Scientists of China (81802735), Beijing Youth Talent Plan (QML20191101), and Science Foundation of Peking University Cancer Hospital (2020-11). The funders had no role in study design, data collection and analysis, decision to publish, or preparation of the manuscript.

\section{Availability of data and materials}

The datasets used and/or analysed during the current study are available from the corresponding author on reasonable request.

\section{Ethics approval and consent to participate}

The study was approved by the Ethics Committee of Peking University Cancer Hospital and the patients' written consent was also obtained. Written informed consent for publication was obtained and stored in Peking University Cancer Hospital.

\section{Consent for publication}

Not applicable.

\section{Competing interests}

The authors declare that they have no competing interests.

Received: 8 May 2020 Accepted: 9 August 2020

Published online: 18 August 2020

\section{References}

1. Bray F, Ferlay J, Soerjomataram I, Siegel RL, Torre LA, Jemal A. Global cancer statistics 2018: GLOBOCAN estimates of incidence and mortality worldwide for 36 cancers in 185 countries. CA Cancer J Clin. 2018;68(6):394-424.

2. Matsubayashi H, Takagaki S, Otsubo T, liri T, Kobayashi Y, Yokota T, et al. Advanced gastric glandular-endocrine cell carcinoma with 1-year survival after gastrectomy. Gastric Cancer. 2000;3(4):226-33.

3. Park JY, Ryu MH, Park YS, Park HJ, Ryoo BY, Kim MG, et al. Prognostic significance of neuroendocrine components in gastric carcinomas. Eur J Cancer. 2014;50(16):2802-9.

4. La Rosa S, Inzani F, Vanoli A, Klersy C, Dainese L, Rindi G, et al. Histologic characterization and improved prognostic evaluation of 209 gastric neuroendocrine neoplasms. Hum Pathol. 2011:42(10):1373-84.

5. Ishida M, Sekine S, Fukagawa T, Ohashi M, Morita S, Taniguchi H, et al. Neuroendocrine carcinoma of the stomach: morphologic and immunohistochemical characteristics and prognosis. Am J Surg Pathol. 2013:37(7):949-59.

6. Rayhan N, Sano T, Qian ZR, Obari AK, Hirokawa M. Histological and immunohistochemical study of composite neuroendocrine-exocrine carcinomas of the stomach. J Med Investig. 2005;52(3-4):191-202

7. Jiang SX, Mikami T, Umezawa A, Saegusa M, Kameya T, Okayasu I. Gastric large cell neuroendocrine carcinomas: a distinct clinicopathologic entity. Am J Surg Pathol. 2006;30(8):945-53.

8. Ohike NAN, La Rosa $\mathrm{S}$, et al. WHO classification of Tumours of endocrine organs. 4th ed. Lyon: IARC Press; 2017.

9. Amin MB, Edge SB. AJCC cancer staging manual: springer; 2017.

10. La Rosa S, Marando A, Sessa F, Capella C. Mixed Adenoneuroendocrine carcinomas (MANECS) of the gastrointestinal tract: an update. Cancers (Basel). 2012;4(1):11-30.

11. La Rosa S, Sessa F, Uccella S. Mixed neuroendocrine-nonneuroendocrine neoplasms (MiNENs): unifying the concept of a heterogeneous Group of Neoplasms. Endocr Pathol. 2016;27(4):284-311.

12. Sano T, Coit DG, Kim HH, Roviello F, Kassab P, Wittekind C, et al. Proposal of a new stage grouping of gastric cancer for TNM classification: international gastric cancer association staging project. Gastric Cancer. 2017;20(2):217-25.

13. Xie JW, Lu J, Wang JB, Lin JX, Chen QY, Cao LL, et al. Prognostic factors for survival after curative resection of gastric mixed adenoneuroendocrine carcinoma: a series of 80 patients. BMC Cancer. 2018;18(1):1021. 
14. Long J, Wang A, Bai Y, Lin J, Yang X, Wang D, et al. Development and validation of a TP53-associated immune prognostic model for hepatocellular carcinoma. EBioMedicine. 2019;42:363-74.

15. de Mestier L, Cros J, Neuzillet C, Hentic O, Egal A, Muller N, et al. Digestive system mixed neuroendocrine-non-neuroendocrine neoplasms. Neuroendocrinology. 2017;105(4):412-25.

16. Rindi G, Arnold R, Bosman F. Nomenclature and classification of neuroendocrine neoplasms of the digestive system. In: WHO classification of Tumours of the digestive system; 2010. p. 13-4.

17. Nishikura K, Watanabe H, Iwafuchi M, Fujiwara T, Kojima K, Ajioka Y. Carcinogenesis of gastric endocrine cell carcinoma: analysis of histopathology and p53 gene alteration. Gastric Cancer. 2003;6(4):203-9.

18. Li Y, Yau A, Schaeffer D, Magliocco A, Gui X, Urbanski S, et al. Colorectal glandular-neuroendocrine mixed tumor: pathologic spectrum and clinical implications. Am J Surg Pathol. 2011;35(3):413-25.

19. Karkouche R, Bachet JB, Sandrini J, Mitry E, Penna C, Cote JF, et al. Colorectal neuroendocrine carcinomas and adenocarcinomas share oncogenic pathways. A clinico-pathologic study of 12 cases. Eur J Gastroenterol Hepatol. 2012;24(12):1430-7.

20. Kim KM, Kim MJ, Cho BK, Choi SW, Rhyu MG. Genetic evidence for the multi-step progression of mixed glandular-neuroendocrine gastric carcinomas. Virchows Arch. 2002;440(1):85-93.

21. Vortmeyer AO, Lubensky IA, Merino MJ, Wang CY, Pham T, Furth EE, et al. Concordance of genetic alterations in poorly differentiated colorectal neuroendocrine carcinomas and associated adenocarcinomas. J Natl Cancer Inst. 1997;89(19):1448-53.

22. Furlan D, Cerutti R, Genasetti A, Pelosi G, Uccella S, La Rosa S, et al. Microallelotyping defines the monoclonal or the polyclonal origin of mixed and collision endocrine-exocrine tumors of the gut. Lab Investig. 2003;83(7):963-71.

23. Scardoni M, Vittoria E, Volante M, Rusev B, Bersani S, Mafficini A, et al. Mixed adenoneuroendocrine carcinomas of the gastrointestinal tract: targeted next-generation sequencing suggests a monoclonal origin of the two components. Neuroendocrinology. 2014;100(4):310-6.

24. Vanacker L, Smeets D, Hoorens A, Teugels E, Algaba R, Dehou MF, et al. Mixed adenoneuroendocrine carcinoma of the colon: molecular pathogenesis and treatment. Anticancer Res. 2014;34(10):5517-21.

25. Travis WD. Pathology \& Genetics Tumours of the lung, pleura, thymus and heart. In: World Health Organization classification of Tumours; 2004.

26. Cunningham D, Allum WH, Stenning SP, Thompson JN, Van de Velde CJ, Nicolson $\mathrm{M}$, et al. Perioperative chemotherapy versus surgery alone for resectable gastroesophageal cancer. N Engl J Med. 2006;355(1):11-20.

27. Chen X, Liu H, Li G, Yu J. Implications of clinical research on adjuvant chemotherapy for gastric cancer: where to go next? Chin J Cancer Res. 2019;31(6):892-900.

28. Ma F, Wang B, Xue L, Kang W, Li Y, Li W, et al. Neoadjuvant chemotherapy improves the survival of patients with neuroendocrine carcinoma and mixed adenoneuroendocrine carcinoma of the stomach. J Cancer Res Clin Oncol. 2020;146(8):2135-42.

29. van der Veen A, Seesing MFJ, Wijnhoven BPL, de Steur WO, van Berge Henegouwen Ml, Rosman C, et al. Management of resectable esophageal and gastric (mixed adeno) neuroendocrine carcinoma: a nationwide cohort study. Eur J Surg Oncol. 2018;44(12):1955-62.

\section{Publisher's Note}

Springer Nature remains neutral with regard to jurisdictional claims in published maps and institutional affiliations.

Ready to submit your research? Choose BMC and benefit from:
- fast, convenient online submission
- thorough peer review by experienced researchers in your field
- rapid publication on acceptance
- support for research data, including large and complex data types
- gold Open Access which fosters wider collaboration and increased citations
- maximum visibility for your research: over 100M website views per year
At BMC, research is always in progress.
Learn more biomedcentral.com/submissions

